\title{
Effects of Short Time Variation in the River Discharge on the Salt Wedge Intrusion in the Yura Estuary, a Micro Tidal Estuary, Japan
}

\author{
Tatsuhiro Funahashi ${ }^{1}$, Akihide Kasai ${ }^{1}$, Masahiro Ueno ${ }^{2}$, Yoh Yamashita ${ }^{2}$ \\ ${ }^{1}$ Graduate School of Agriculture, Kyoto University, Kyoto, Japan \\ ${ }^{2}$ Field Science Education and Research Center, Kyoto University, Maizuru, Japan \\ Email: tatuhiro@kais.kyoto-u.ac.jp
}

Received December 25, 2012; revised January 26, 2013; accepted February 7, 2013

\begin{abstract}
A numerical model was developed to investigate salinity distribution in the Yura Estuary, a micro tidal estuary in Japan. The model results show that the salinity distribution as represented by salt wedge intrusion agreed well with field observations. In addition to the seasonal variation, the salt wedge responds over short time scales according to the flood events. The retreat of the salt wedge is dependent on the scale of the river discharge; the salt wedge moved back and disappeared from the estuary when over $250 \mathrm{~m}^{3} \cdot \mathrm{s}^{-1}$ of fresh water was discharged from the estuary and it takes $\sim 11$ days for salt wedge to recover from the fresh water discharge event. The Yura Estuary has on average three floods during summer, this coincides with when phytoplankton is most productive in the river and indicates that the short temporal variations in the river discharge has important effects not only on the hydrodynamics, but also on the ecosystem in the estuary.
\end{abstract}

Keywords: Micro Tidal Estuary; River Discharge; Salt Wedge Intrusion; Short Time Variation

\section{Introduction}

Estuaries are formed around the river mouths, where lighter fresh water meets denser sea water. There is a great deal of variety in mixing processes and salinity distributions, affected by the balance between tides and river discharges [1]. For instance, during periods of strong tidal forces and low runoff, the Columbia River Estuary becomes well mixed, where salinity structure is vertically homogeneous [2]. However, estuaries along the Mediterranean coast are known as highly stratified estuaries, where sharp vertical salinity gradients are observed because of their small tidal ranges [3]. Reference [3] categorized highly stratified estuaries into two types based on the river discharge, estuarine cross section, and tidal range: 1) the salt wedge regime is established during low discharge periods, whereas it is washed away during high river discharge; 2) The salt wedge regime is established during high river discharge periods, but the water is partially mixed and the obvious salt wedge is not observed during low discharge periods.

Fresh water is utilized for irrigation and drinking purposes in many rivers. Therefore, salt wedge intrusions have been studied for management of estuarine water quality [4]. Reference [5] used a two-layer hydrodynamic model to evaluate effects of the change in the river discharge on the salt wedge dynamics of the Ebro River estuary, which belongs to the first type of highly stratified estuary according to Ibañez's classification [3]. Model results suggest that the decrease in the river discharge enlarges and prolongs the salt wedge. However, these studies dealt with long term (more than few months) trends, and there are few studies on the short term variation in the slat wedge intrusion in micro tidal estuaries.

Compared to American and Southeastern Asian continental countries, Japan is characterized by a relatively narrow strip of mountainous land. This implies that the root of its rivers bear a strong altitude gradient from the origin to the mouth, leading to short residence time of rainwater, as well as strong and rapid variations in river discharge [6]. These sudden changes in turn impact estuarine dynamics. This study was conducted in the Yura Estuary, the first type of micro tidal estuary according to Ibañez's classification, which has the salt wedge in summer. A previous study revealed that the salt wedge in the Yura River changes seasonally and is affected by variations in river discharge and sea level [7]. It is also shown that a chlorophyll maximum was generated around a halocline during summer salt wedge regime, indicating that the salt wedge plays an important role in 
the ecology of the Yura Estuary. During summer, discharge of Yura River also varies drastically over short time scales due to heavy rains. In this study, therefore, we evaluate the effect of short temporal variations in the river discharge on the salt wedge intrusion in the Yura Estuary using a three dimensional numerical model. First, the model was developed to reproduce the real salt wedge intrusion, which was validated with field observations [7]. The model was then applied to an experiment that examines the response of the salt wedge to the rapid change in the river discharge in the Yura Estuary as representative of micro tidal estuaries.

\section{Materials and Methods}

\subsection{Study Site}

The Yura River is a $146 \mathrm{~km}$ long and flows into Wakasa bay, Sea of Japan (Figure 1). The catchment area is about $1880 \mathrm{~km}^{2}$. The mean annual discharge measured at Fukuchiyama is $\sim 51.5 \mathrm{~m}^{3} \cdot \mathrm{s}^{-1}$ in the last 54 years. During winter, northwesterly winds off the Asian continent bring heavy snow on Sea of Japan side. The average river discharge is large $\left(\sim 55 \mathrm{~m}^{3} \cdot \mathrm{s}^{-1}\right)$ in winter and early spring because of snow melting. However, the average discharge decreases to $21 \mathrm{~m}^{3} \cdot \mathrm{s}^{-1}$, because summer to autumn is relatively dry season. The catchment area is still subjected to sudden heavy rain in summer so that the discharges sometimes reach over $100 \mathrm{~m}^{3} \cdot \mathrm{s}^{-1}$. Therefore the salt wedge intrusion varies seasonally [7], and it is expected to change suddenly in summer.

The typical tidal range of the Yura Estuary is less than $0.5 \mathrm{~m}$, and it is thus classified as a micro tidal estuary. Therefore the effects of tidal currents on the physical conditions are negligible. The Yura Estuary enters the salt wedge regime during low discharge periods, with a maximum salt wedge length of $18 \mathrm{~km}$ from the river mouth [7]. The estuarine width is $100 \mathrm{~m}$ at the upper part and increases to $500 \mathrm{~m}$ at the mouth. Average water depth is $3.7 \mathrm{~m}$ and there is a deep part $(\sim 10 \mathrm{~m})$ at $5-6$ $\mathrm{km}$ from the mouth.

\subsection{Numerical Model}

\subsubsection{Model Design}

The salt wedge dynamics were calculated by Delft3DFlow [8]. This model is a three dimensional hydrodynamic model developed by Delft Hydraulics. The model domain extends $25 \mathrm{~km}$ from the upper part of the Yura Estuary to $3 \mathrm{~km}$ offshore from the river mouth (Figures 1 and 2). Curvilinear grids were applied for the width of river part domain so that the model domain corresponds to the real topography. The original bathymetry data of the upper Yura estuarine part were obtained from the Ministry of Land, Infrastructure, Transport and Tourism,

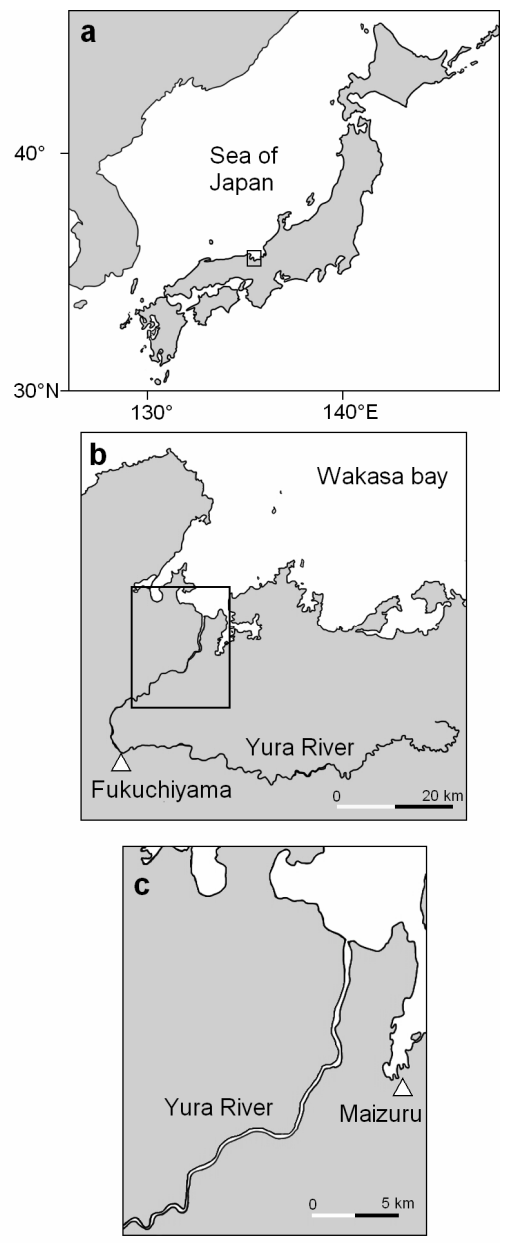

Figure 1. Location and bathymetry of the Yura Estuary.

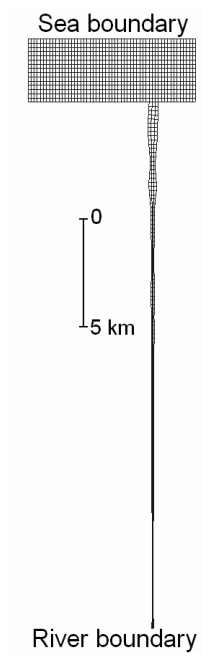

Figure 2. Model domain and curvilinear grids used in the simulations.

and the coastal area from the Japan Coast Guard. The model system has $14 \sigma$-levels in the vertical; five layers of $3 \%$, three of $5 \%$, four of $15 \%$, two of $5 \%$ from the 
surface to bottom. The horizontal grid scale ranges from $15 \mathrm{~m}$ by $200 \mathrm{~m}$ in the river to $150 \mathrm{~m}$ by $200 \mathrm{~m}$ in the sea. The vertical eddy viscosity and diffusivity are calculated by a $k-\varepsilon$ model. The horizontal eddy viscosity and diffusivity are calculated by a 3D-turbulence closure model [8]. Both the river end $(y=0 \mathrm{~km})$ and the sea side $(y=28$ $\mathrm{km}$ ) are open boundaries. Monthly observations of salinity and temperature [7] are used to force the open boundary. Daily averaged sea level at Maizuru and daily average river discharge at Fukuchiyama are also used for the sea side open boundary and river side open boundary, respectively (Figures $\mathbf{2}$ and $\mathbf{3}$ ).

The salt wedge intrusion was simulated for two years; from April 2006 to March 2008. The model was spun up and reached steady state after one month, and then real calculation started with observed boundary conditions. Calculated salinity distribution and salt wedge length by the model were compared with those obtained by field observations [7]. In this study, salt wedge length is defined as the distance from the river mouth to the tip of salt wedge, which salinity is 10 at the bottom.

\subsubsection{Responses of the Salt Wedge to the Flood}

In order to study the response of the salt wedge to variations in river discharge, the river discharge condition was changed while the boundary conditions of temperature, salinity and sea level were kept constant. With the intention of reproduce the summer salinity distribution, the
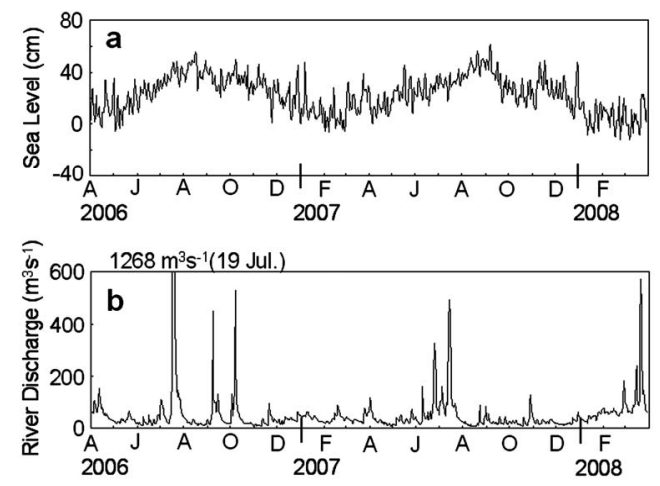

Figure 3. Time series of (a) Sea level in Maizurum, and (b) River discharge in Fukuchiyama.

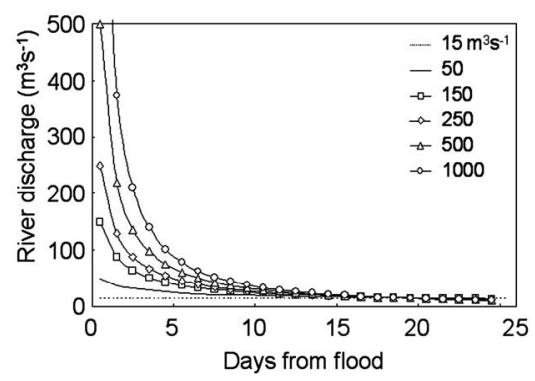

Figure 4. Time series of river discharge with each scenarios of $Q_{0}$. salinity and temperature of the river open boundary were set to 0 and $15.4^{\circ} \mathrm{C}$ respectively, and the sea side boundary conditions were set to $33.4,17.6^{\circ} \mathrm{C}$ and $0.2 \mathrm{~m}$ for salinity, temperature and sea level condition respectively. The river discharge $(Q)$ of a flood event was empirically calculated as follows;

$$
Q=Q_{0} T^{\left\{-0.34 \ln \left(Q_{0}\right)+0.93\right\}},
$$

where $Q_{0}$ represents maximum discharge $\left(\mathrm{m}^{3} \cdot \mathrm{s}^{-1}\right)$ and $T$ is date from the flood event (day). Equation (1) is derived from typical flood event conditions and river flow decreases gradually without any increasing of the river discharge after the flood.

First, the model was run for one month with a constant river discharge $\left(15 \mathrm{~m}^{3} \cdot \mathrm{s}^{-1}\right)$ in order to obtain a steady state as representative for dry season. The second stage of the experiment was forced with the steady state as the initial condition and then the river discharge was changed according to Equation (1). Eight flood scenarios of $Q_{0}$ equals 50, 100, 150, 200, 250, 500, 1000 and 1500 were conducted. The minimum river discharge was set to $15 \mathrm{~m}^{3} \cdot \mathrm{s}^{-1}$ Examples of variation of discharge are 50, 150, 250, 500 and $1000 \mathrm{~m}^{3} \cdot \mathrm{s}^{-1}$ are shown in Figure 4. This shows that the difference of $Q$ among the scenarios was large in the early stages, but reduced to $15 \mathrm{~m}^{3} \cdot \mathrm{s}^{-1} 20$ days after the flood regardless of $Q_{0}$. This is consistent with the observed river discharge at Fukuchiyama. The response of the salt wedge was evaluated by comparing the minimum salt wedge length and replacing time, which represents the time that takes salt wedge to re-intrude from the minimum position to $12.5 \mathrm{~km}\left(\mathrm{~T}_{12.5}\right)$ and $15 \mathrm{~km}$ $\left(T_{15}\right)$. In this study, the minimum salt wedge length is defined as the minimum position during the experiment.

\section{Results}

\subsection{Reproduction of the Observed Salinity Distribution}

Figure 5 shows the salinity distributions obtained by field observations and model simulations. The river discharge was low on 11 December 2006 and 22 August 2007, while high on 29 January 2008 and 25 February 2008. The salt wedge intruded into the river, which was strongly stratified under low river discharge conditions (Figures 5(a) and (c)). The upper fresh water layer was thicker in December 2006 than that in August 2007, depending on the river discharge and sea level (Figure 3), although the salt wedge intruded into the river in both dates. The model reproduced the salinity distribution well, showing the salt wedge intrusion through the bottom layer with the strong halocline (Figures 5(b) and (d)). In contrast, the river was occupied by the fresh water and the salt wedge was washed away on 29 January and 25 February 2008 (Figures 5(f) and (h)). Slightly 


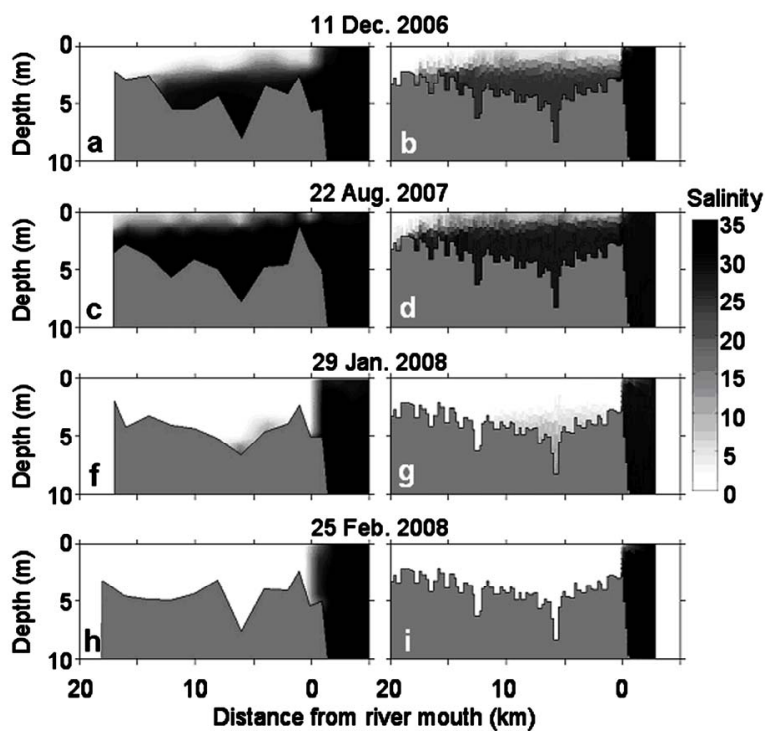

Figure 5. Salinity distributions in the Yura River. Left column shows observed results and right shows calculated results.

salty water (salinity less than 10) was arrested around 5 $\mathrm{km}$ from the river mouth in January 2008. The salinity distributions by the model correspond well to the field observations under the high river discharge conditions (Figures 5(g) and (i)).

Figure 6 shows time change in salt wedge length obtained by the model and field observations. Observed salt wedge length decreased during high river discharge season, January-April when melted snow flows into river and June-July when the river discharge increased by sudden heavy rain (Figure 3(b)). On the other hand, the salt wedge length increased in August-December when the river discharge was low. In addition, even when the river discharge was low, salt wedge lengths in November-May were shorter than those in June-October probably because of the low sea level. The calculated salt wedge length retreated significantly in April 2006 and January-March 2008. Among the three snow melting periods, the salt wedge intrudes up to $15 \mathrm{~km}$ in the beginning of 2007 (Figure 6(a)), when the precipitation was relatively small (Figure 3). These results are consistent with the observed intrusion. In comparison, the large floods pushed salt wedge back in July 2006 and 2007. The observational and model results correspond well each other. From August to December, the modeled salt wedge remained relatively long, except in September and October 2006, when a sudden run off washed the salt wedge away. The overall calculated salt wedge lengths were correlated well with the observed lengths $\left(\mathrm{r}^{2}=0.59\right)$. This indicates the model can reliably simulate the real salinity distribution in the Yura Estuary.

It is worth noticing that the model results show not only the seasonal variation of the salt wedge, but also the short term variations (Figure 6(a)). The simulated salt wedge shows retreats and re-intrusions in May-July 2006, September-October 2006 and January-April 2007, corresponding to variations in the river discharge (Figures 3 and 6). In addition, small fluctuations were recognized in May and August-October 2007. The amplitude of fluctuations ranged from 0.2 to $7 \mathrm{~km}$ with an average of $1.1 \mathrm{~km}$ (Figure 6(b)). These short time variations cannot be detected by the monthly field observations. Therefore it is a significant advantage of the numerical model that it can identify the high resolution temporal and spatial variations.

\subsection{Responses of the Salt Wedge to the Flood}

The responses of the salt wedge to the flood correspond to the scale of the river discharge (Figure 7). The larger $Q_{0}$ leads to a smaller intrusion of the salt wedge. When $Q_{0}$ equaled more than $250 \mathrm{~m}^{3} \cdot \mathrm{s}^{-1}$ the salt wedge is extruded. In addition, Figure 8 shows that a larger $Q_{0}$ prolonged the recovery time. However, it reached a threshold
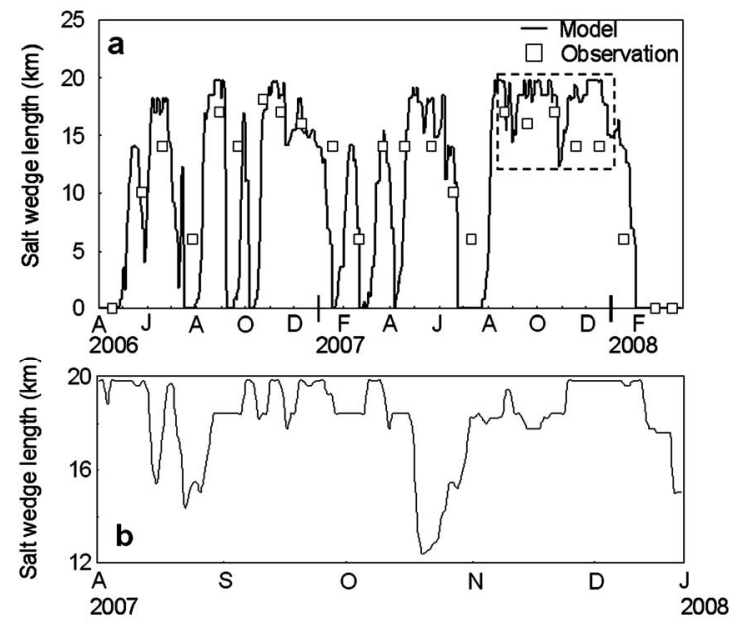

Figure 6. (a) Time series of salt wedge length. Solid line shows daily salt wedge length obtained by the model, and open square $(\square)$ shows monthly ones obtained by the observations; (b) A part of time series of salt wedge length ranged from 12 to $20 \mathrm{~km}$. Time series in (b) corresponds to dashed square part in (a).

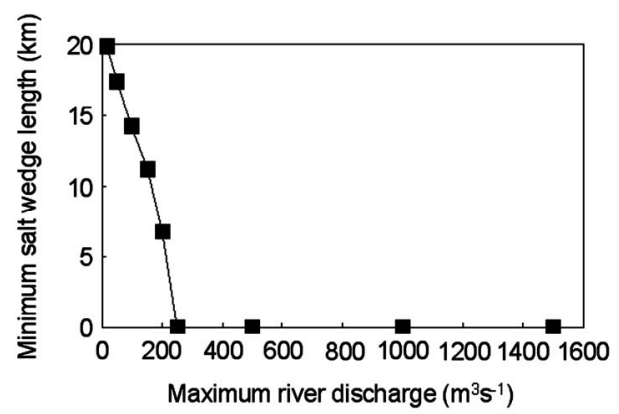

Figure 7. Relation between minimum salt wedge length and maximum river discharge calculated by the model. 


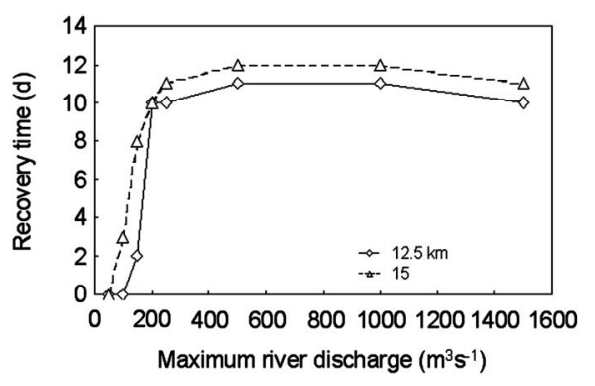

Figure 8. Variation in the recovery time with each flood scenario. Open diamonds $(\diamond)$ with solid line show T12.5, and open triangles $(\triangle)$ with broken line shows $T 15$.

old limit around 11 days with over $250 \mathrm{~m}^{3} \cdot \mathrm{s}^{-1}$ of $Q_{0}$. This corresponds to the length of the salt wedge (Figure 7), indicating that it takes $\sim 11$ days for the salt wedge to re-intrude into the river and approach the maximum position after the flush out event. The phase speed $(c)$ of the intrusion of the bottom dense water in two layers system is given by

$$
c=\sqrt{\frac{\Delta \rho}{\rho} g H},
$$

where $\Delta \rho$ is the difference in density between the two layers, $\rho$ the averaged density of water, $g$ the acceleration of gravity, and $H$ the height of the lower layer. $\Delta \rho, \rho$ and $H$ were $15 \mathrm{~kg} \cdot \mathrm{m}^{-3}, 1001 \mathrm{~kg} \cdot \mathrm{m}^{-3}$ and $0.33 \mathrm{~m}$ respectively, at $200 \mathrm{~m}$ from the river mouth when the salt wedge started to re-intrude into the river in case of $Q_{0}=$ $1000 \mathrm{~m}^{3} \cdot \mathrm{s}^{-1}$. The phase speed $(c)$ is thus $22 \mathrm{~cm} \cdot \mathrm{s}^{-1}$. This value corresponds to the modeled speed of salt wedge intrusion, which is calculated as 11 days to reach $15 \mathrm{~km}$ upstream of the river mouth.

\section{Discussion}

The length of the salt wedge in the Yura Estuary increases in the summer and autumn, while decreases in the winter and spring [7]. In this study, our model can reproduce the seasonal variation in longitudinal salinity distributions and then simulate the salt wedge intrusion into the estuary (Figures $\mathbf{4}$ and 5). In addition, the model results show the short term variations most likely due to variations of the river discharge. The Yura River, like the other rivers in Japan, experiences drastic flood events in summer (Table 1 and Figure 3(b)). The model shows that a flood washes the salty bottom water away from the estuary (e.g. August and September 2006, and July 2007).

When the river discharge was less than $100 \mathrm{~m}^{3} \cdot \mathrm{s}^{-1}$, the short term variation in salt wedge length was $0.2-7 \mathrm{~km}$ (Figure 6(b)). Considering that the maximum salt wedge length is $\sim 18 \mathrm{~km}$, this daily variation is relatively small. Reference [3] showed that the salt wedge length changed
Table 1. Annual frequencies of $\geq 200 \mathrm{~m}^{3} \cdot \mathrm{s}^{-1}$ flood from May to October.

\begin{tabular}{ll}
1990 & 4 \\
1991 & 2 \\
1992 & 2 \\
1993 & 3 \\
1994 & 1 \\
1995 & 2 \\
1996 & 3 \\
1997 & 3 \\
1998 & 4 \\
1999 & 7 \\
2000 & 1 \\
2001 & 3 \\
2002 & 1 \\
2003 & 3 \\
2004 & 7 \\
2005 & 1 \\
2006 & 3 \\
2007 & 2 \\
Average & 2.9 \\
\hline
\end{tabular}

little when the river discharge varied from 50 to 160 $\mathrm{m}^{3} \cdot \mathrm{s}^{-1}$ within 24 hours in the Ebro Estuary, a micro tidal estuary in Spain. It was also reported in the Strymon Estuary, a micro tidal estuary in Greece, that the distance of the salt wedge moved only $2 \mathrm{~km}$ when the river discharge varied from 34 to $49 \mathrm{~m}^{3} \cdot \mathrm{s}^{-1}$ within 10 days [9]. In comparison, the salt wedge in Tweed Estuary (a meso tidal estuary in the United Kingdom) varied $2-5 \mathrm{~km}$ within a semidiurnal time scale affected by variation of water level [10]. The length of the salt wedge changes $3.7 \mathrm{~km}$ in spring tide and $3.0 \mathrm{~km}$ in neap tide in the lower part of Naka River, where the tidal range is $\sim 1.5 \mathrm{~m}$ and salt water intrudes $17.5 \mathrm{~km}$ at most [11]. Together with the previous studies, our results indicate that the salt wedge is more stable in the micro tidal estuaries than in the meso and macro tidal estuaries under the small discharge condition.

The flood event with the river discharge of $100 \mathrm{~m}^{3} \cdot \mathrm{s}^{-1}$ pushes salt wedge back $3 \mathrm{~km}$ (Figure 7). Salt water is washed away when the peak of the discharge exceeds $250 \mathrm{~m}^{3} \cdot \mathrm{s}^{-1}$ (Figure 7). The Yura River has $1-7$ flood events which discharge more than $200 \mathrm{~m}^{3} \cdot \mathrm{s}^{-1}$ in MayOctober, when phytoplankton is abundant in the estuary. This indicates that the Yura Estuary is often refreshed during high productive season. As the chlorophyll maximum is generated around the halocline during summer salt wedge regime [7], frequent flushing events would play an important role in the ecosystem in the Yura Es- 
tuary.

It was also reported that salt intrusion affected phytoplankton blooming in other highly stratified estuary [12]. However, the detailed production mechanism is still unclear in relation to the salt wedge. It is therefore important to reveal the processes and relationship between the salty water intrusion and primary production in the estuary. One of the useful methods to understand the mechanism might be the direct measurement of the phytoplankton production in the estuary. However, this method is unsatisfied to examine the temporal variations in the production related to the salt wedge movement, although it can measure the real production in the field. Instead we suggest an ecological and hydrodynamic coupled modeling approach would be more suitable for examining short time change in primary production in future studies.

\section{Acknowledgements}

This study was partly supported by the Coastal Ecosystem Complex Project of the Ministry of Education (ID number: 21380122).

\section{REFERENCES}

[1] K. R. Dyer, "Estuaries, a Physical Introduction," 2nd Edition, John Wiley \& Sons Ltd., Hoboken, 1997.

[2] D. A. Jay and J. Dungan Smith, "Circulation, Density Distribution and Neap-Spring Transitions in the Columbia River Estuary," Progress in Oceanography, Vol. 25, No. 1-4, 1990, pp. 81-112. doi:10.1016/0079-6611(90)90004-L

[3] C. Ibañez, D. Pont and N. Part, "Characterization of the Ebre and Rhone estuaries: A Basis for Defining and Classifying Salt-Wedge Estuaries," Limnology and Oceanog- raphy, Vol. 42, No. 1, 1997, pp. 89-101. doi:10.4319/10.1997.42.1.0089

[4] H. H. S. Savenije, "Predictive Model for Salt Intrusion in Estuaries," Journal of Hydrology, Vol. 148, No. 1-4, 1993, pp. 203-218. doi:10.1016/0022-1694(93)90260-G

[5] J. P. Sierra, A. Sánchez-arcilla, P. A. Figueras, J. González Del Río, E. K. Rassmussen, C. Mösso, "Effects of Discharge Reductions on Salt Wedge Dynamics of the Ebro River," River Research and Applications, Vol. 20, No. 1, 2004, pp. 61-77. doi:10.1002/rra.721

[6] S. Unoki, "The Science of Flow System," Tsukiji Shokan Publishing, Tokyo, 2010, p. 99.

[7] A. Kasai, Y. Kurikawa, M. Ueno, D. Rober and Y. Yamashita, "Salt-Wedge Intrusion of Seawater and Its Implication for Phytoplankton Dynamics in the Yura Estuary, Japan," Estuarine, Coastal and Shelf Science, Vol. 86, No. 3, 2010, pp. 408-414. doi:10.1016/j.ecss.2009.06.001

[8] Delft Hydraulics, "Delft3D-FLOW User Manual Version 3.14,” Delft, 2007.

[9] K. Harambidou, G. Sylaios and V. A. Tsihrintzis, "SaltWedge Propagation in a Mediterranean Micro-Tidal River Mouth," Estuarine, Coastal and Shelf Science, Vol. 90, No. 4, 2010, pp. 174-184. doi:10.1016/j.ecss.2010.08.010

[10] R. J. Uncles and J. A. Stephens, "Salt Intrusion in the Tweed Estuary," Estuarine, Coastal and Shelf Science, Vol. 43, No. 3, 1996, pp. 271-293. doi:10.1006/ecss.1996.0070

[11] S. Fukuoka, H. Shimamura, Y. Kajiya, A. Takahashi and K. Okada, "Field Study of Salt Intrusion in Naka River," Proceedings of the Japanese Conference on Hydraulics, Vol. 32, No. 1, 1988, pp. 203-208.

[12] P. A. Thompson, "Spatial and Temporal Patterns of Factors Influencing Phytoplankton in a Salt wedge Estuary, the Swan River, Western Australia," Estuaries, Vol. 21, No. 4, 1998, pp. 801-817. doi:10.2307/1353282 